\title{
BMJ Open Comparing docosahexaenoic acid (DHA) concomitant with neoadjuvant chemotherapy versus neoadjuvant chemotherapy alone in the treatment of breast cancer (DHA WIN): protocol of a double-blind, phase II, randomised controlled trial
}

Marnie Newell, ${ }^{1}$ John R Mackey, ${ }^{2,3}$ Gilbert Bigras, ${ }^{4}$ Mirey Alvarez-Camacho, ${ }^{3}$ Susan Goruk, ${ }^{1}$ Sunita Ghosh, ${ }^{3}$ Alison Schmidt, ${ }^{3}$ Deborah Miede, ${ }^{3}$ Ann Chisotti, ${ }^{3}$ Lynne Postovit, ${ }^{2}$ Kristi Baker, ${ }^{2}$ Vera Mazurak, ${ }^{1}$ Kerry Courneya, ${ }^{5}$ Richard Berendt, ${ }^{4}$ Wei-Feng Dong, ${ }^{4}$ George Wood, ${ }^{4}$ Sanraj K Basi, ${ }^{3}$ Anil Abraham Joy, ${ }^{2}$ Karen King, ${ }^{3}$ Judith Meza-Junco, ${ }^{3}$ Xiaofu Zhu, ${ }^{3}$ Catherine Field ${ }^{1}$

To cite: Newell M, Mackey JR, Bigras G, et al. Comparing docosahexaenoic acid (DHA) concomitant with neoadjuvant chemotherapy versus neoadjuvant chemotherapy alone in the treatment of breast cancer (DHA WIN): protocol of a double-blind, phase II, randomised controlled trial. BMJ Open 2019;9:e030502. doi:10.1136/ bmjopen-2019-030502

- Prepublication history and additional material for this paper are available online. To view these files, please visit the journal online (http://dx.doi. org/10.1136/bmjopen-2019030502).

Received 18 March 2019 Revised 09 August 2019 Accepted 16 August 2019

Check for updates

(c) Author(s) (or their employer(s)) 2019. Re-use permitted under CC BY-NC. No commercial re-use. See rights and permissions. Published by BMJ.

For numbered affiliations see end of article.

Correspondence to

Dr Catherine Field;

catherine.field@ualberta.ca

\section{ABSTRACT}

Introduction Neoadjuvant chemotherapy for breast cancer treatment is prescribed to facilitate surgery and provide confirmation of drug-sensitive disease, and the achievement of pathological complete response (pCR) predicts improved long-term outcomes. Docosahexaenoic acid (DHA) has been shown to reduce tumour growth in preclinical models when combined with chemotherapy and is known to beneficially modulate systemic immune function. The purpose of this trial is to investigate the benefit of DHA supplementation in combination with neoadjuvant chemotherapy in patients with breast cancer. Methods and analysis This is a double-blind, phase II, randomised controlled trial of 52 women prescribed neoadjuvant chemotherapy to test if DHA supplementation enhances chemotherapy efficacy. The DHA supplementation group will take $4.4 \mathrm{~g} /$ day DHA orally, and the placebo group will take an equal fat supplement of vegetable oil. The primary outcome will be change in Ki67 labelling index from prechemotherapy core needle biopsy to definitive surgical specimen. The secondary endpoints include assessment of (1) DHA plasma phospholipid content; (2) systemic immune cell types, plasma cytokines and inflammatory markers; (3) tumour markers for apoptosis and tumour infiltrating lymphocytes; (4) rate of pCR in breast and in axillary nodes; (5) frequency of grade 3 and 4 chemotherapy-associated toxicities; and (6) patient-perceived quality of life. The trial has $81 \%$ power to detect a significant between-group difference in Ki67 index with a two-sided t-test of less than 0.0497, and accounts for $10 \%$ dropout rate.

Ethics and dissemination This study has full approval from the Health Research Ethics Board of Alberta Cancer Committee (Protocol \#: HREBA.CC-18-0381). We expect to present the findings of this study to the

\section{Strengths and limitations of this study}

- This study is the first phase II, randomised controlled trial to evaluate docosahexaenoic acid (DHA) supplementation concomitant with neoadjuvant chemotherapy to treat non-metastatic breast cancer.

- The intervention is minimally invasive and side effects from the supplementation are not expected.

- This study is powered to examine the key clinical outcome of changes in Ki67 index from prechemotherapy biopsy to surgical excision based on group sample sizes of 23 patients in group 1 and 23 patients in group 2 in order to achieve $81 \%$ power to detect a difference between the group proportions of 0.4 .

- This study will measure clinically relevant intermediate outcomes, including rate of pCR in breast and in axillary nodes, rate of grade 3 and 4 chemotherapy-associated toxicities and hospitalisations, as well as additional outcomes including plasma phospholipid content of DHA, markers of immune function (plasma cytokines, inflammatory markers and lymphocyte function), tumour markers for apoptosis and tumour infiltrating lymphocytes, and patient-perceived quality of life.

- The study will include all subtypes of patients with breast cancer undergoing neoadjuvant chemotherapy, but is not powered to assess differences between subtypes.

scientific community in peer-reviewed journals and at conferences. The results of this study will provide evidence for supplementing with DHA during neoadjuvant chemotherapy treatment for breast cancer. Trial registration number NCT03831178 


\section{INTRODUCTION}

Despite improvements in early diagnosis and treatment, breast cancer remains the second leading cause of cancer-related death in women. ${ }^{1}$ While neoadjuvant chemotherapy aims to improve surgical resection outcomes and reduce/eliminate micrometastases, ${ }^{2} 3$ pathological complete response (pCR) is not achieved by all patients. ${ }^{3}$ Increasing the efficacy of neoadjuvant treatment without adding additional side effects would benefit this population.

Docosahexaenoic acid (DHA) is an omega-3 long-chain polyunsaturated fatty acid (n-3 LCPUFA). The majority of n-3 fatty acids are in the form of the 18-carbon fatty acid alpha-linolenic acid (ALA). While DHA can be synthesised from ALA and other n-3 LCPUFA in the body, endogenous synthesis is low. ${ }^{45}$ Consequently, the direct consumption of this fatty acid is the only way to significantly increase levels of DHA in tissues. ${ }^{6}$ Supplementation can increase blood plasma DHA concentration by twofold $(500 \mu \mathrm{M})$, which can lead to plasma membrane lipid enrichment. ${ }^{7}$ Incorporation of DHA into tumour membrane phospholipids has been shown to reduce breast cancer cell proliferation $^{89}$ and increase apoptosis ${ }^{10-15}$ in vitro, and to decrease tumour growth in animal models. ${ }^{13}$ 15-17 Additionally, providing/feeding DHA has been shown to increase the efficacy of different chemotherapeutic drugs in animal models of breast cancer. ${ }^{11} 121819$ While there is limited clinical evidence, it has been shown that increased dietary intake of n-3 LCPUFA, including DHA, results in increased DHA incorporation in breast adipose tissue, ${ }^{20}$ and this correlates with improved response to chemotherapy. ${ }^{21}$ In an open-label trial with patients with advanced metastatic breast cancer, DHA supplementation and enrichment into plasma phospholipids were associated with improved outcomes. ${ }^{22}$ Other clinical trials have reported that supplementation with n-3 LCPUFA at a wide range of doses ( $0.6-8.6 \mathrm{~g}$ /day) increased tolerability of chemotherapeutic drugs in a range of malignancies at other sites, including lung, pancreatic and colorectal (reviewed in $\operatorname{ref}^{23}$ ). Consequently, we hypothesise that the therapeutic index (efficacy to toxicity ratio) of neoadjuvant breast cancer chemotherapy will be improved with the addition of DHA to the treatment.

Breast cancer proliferation can be assessed by immunohistochemical (IHC) analysis of cells staining positive for the nuclear antigen $\mathrm{Ki} 67,{ }^{24}$ as it is expressed in all phases of the cell cycle, $G_{1}, S, G_{2}$ and $M$, but not in $G_{0}{ }^{25}{ }^{26}$ The proportion of cells staining for $\mathrm{Ki} 67$ is frequently used as a primary endpoint to measure efficacy of neoadjuvant therapy in clinical trials. The Ki67 index, defining the change between pretreatment and post-treatment Ki67, has been reported to be an independent prognostic factor in luminal A, luminal B, triple-negative and Human Epidermal Growth Factor Receptor 2 (HER2)-positive breast cancer, and has been reported to be a useful surrogate marker of relapse-free survival in luminal B, triple-negative and HER2-positive breast cancer. ${ }^{27} 28$

\section{Objectives}

The objective of this randomised controlled trial is to assess the efficacy of supplemental DHA combined with neoadjuvant chemotherapy in treatment-naïve women with breast cancer measured by changes in Ki67 index from biopsy to surgical excision. We hypothesise that DHA supplementation will increase plasma phospholipid DHA and improve response to neoadjuvant chemotherapy assessed by a decrease in the Ki67 index.

This protocol follows the Standard Protocol Items: Recommendations for Interventional Trials (SPIRIT) guideline (SPIRIT checklist: online supplementary table 1; WHO checklist: online supplementary table 2). ${ }^{29} 30$

\section{Study design}

The Docosahexaenoic acid (DHA) for Women with Breast Cancer in the Neoadjuvant Setting (DHA WIN) trial will be a two-arm, double-blind, phase II, randomised controlled trial comparing DHA supplementation and placebo (vegetable oil). The proposed study design with outcomes depicted is shown in figure 1, and the SPIRIT participant flow chart is shown in figure 2 .

\section{METHODS AND ANALYSIS \\ Study population}

The study will include eligible women with invasive breast cancer (clinical stage I, II or III) for whom systemic chemotherapy $^{31}$ is recommended prior to surgery. The study will occur at the Cross Cancer Institute, with central laboratory and clinical analyses occurring at the University of Alberta, both in Edmonton, Alberta, Canada. Inclusion and exclusion criteria are listed in box 1 .

\section{Intervention}

All women in this trial will receive standard-of-care chemotherapy throughout the duration of the trial. Breast cancer chemotherapy is developed in a guideline-coordinated system by a single team residing at the Cross Cancer Institute. Consequently, there are only two chemotherapy regimens that are used for neoadjuvant chemotherapy in this population. Each of the two regimens is six cycles in length and given at 3-week intervals with a resultant chemotherapy regimen duration of 18 weeks. Both regimens are docetaxel-based. For HER2-negative disease, patients universally receive the FEC-D (fluorouracil, epirubicin, cyclophosphamide; docetaxel) ${ }^{32}$ regimen as neoadjuvant therapy, while HER2-positive patients receive the $\mathrm{DCH}$ regimen (docetaxel, carboplatinum, trastuzumab). ${ }^{33}$

Patients will be prescribed either $4.4 \mathrm{~g}$ /day DHA (in 11-1 g capsules), in the form of DHA-enriched, algaesourced triglyceride oil capsules (life'sDHA S40-O400), or $11 \mathrm{~g}$ placebo (corn/soy oil blend) per day (capsules from DSM Nutritional Products, Columbia, Maryland; online supplementary table 3 for the main fatty acid content of DHA and the placebo). The placebo is balanced for polyunsaturated fatty acid content with linoleic acid to match 


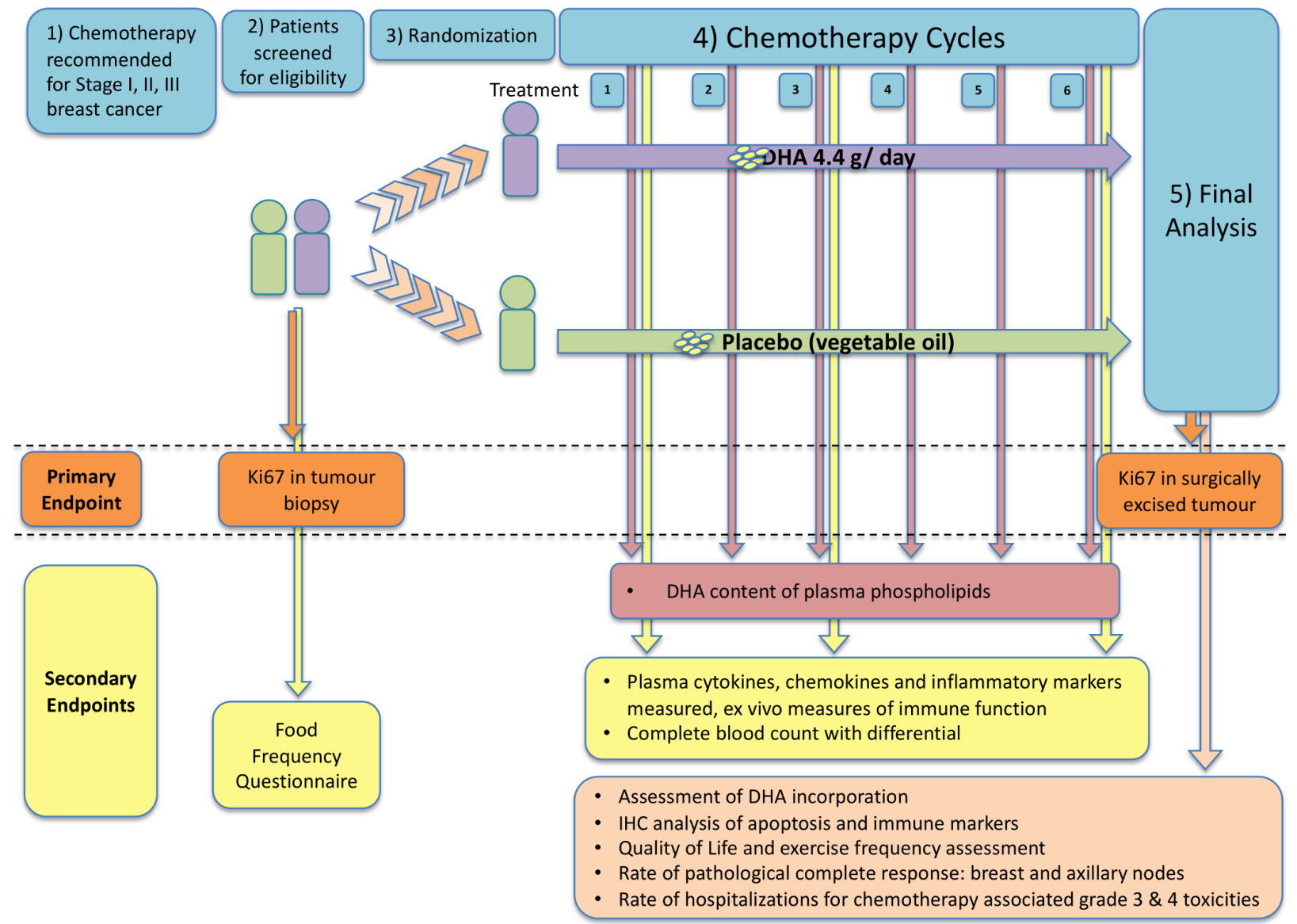

Figure 1 Flow chart of trial design with endpoints and proposed experimental analyses. DHA, docosahexaenoic acid; IHC, immunohistochemistry.

the DHA treatment. The amount of additional linoleic acid in the diet of this group is not expected to increase inflammation $^{34}$ and has not been shown to elicit a tumorous response. ${ }^{35}$ The capsules are to be taken orally throughout the day as tolerated (at any time, with or without food). Capsules are identical in appearance and composition (other than the oils) to maintain blinding of participants and study staff. As the DHA source is an algae-synthesised triglyceride, there are no differences in texture or taste.

All patients will begin a cytotoxic chemotherapy regimen intended to require 18 weeks for delivery. The intervention (DHA or placebo) will commence at the start of the first cycle of chemotherapy and continue through four to six cycles of chemotherapy (3 weeks/ cycle). Should a patient not be able to complete the full six cycles of therapy, the timing of surgery remains $3-5$ weeks after the last cycle of chemotherapy is delivered. As local guidelines mandate surgery between 3 and 5 weeks from the last round of chemotherapy, DHA/placebo will be continued until this time (21-35 days after the last administration of cytotoxic chemotherapy).

All patients will be dispensed an additional bottle of DHA/placebo capsules at the beginning of the study to account for circumstances where their treatment is delayed due to treatment-associated toxicities (including but not limited to vomiting, diarrhoea, abnormalities in blood work, fatigue or severe mouth sores). The patients will be requested to continue taking the DHA or placebo as tolerated and will be dispensed additional capsules as necessary. The extra capsules will remain with the patient until the end of the study.

Patients will be encouraged to take the supplements as tolerated (throughout the day at any time, with or without food). Treatment adherence will be monitored by a review of the patient dosing diary and recording the number of any remaining capsules returned at the end of study visit following the last dose of DHA/placebo. Non-compliance will be assessed as consuming less than $50 \%$ of the weekly dose for two consecutive cycles. No additional natural health product is permitted beyond a daily multivitamin.

\section{Outcome measurements}

Study outcome timelines are summarised in table 1 . Briefly, outcomes will be measured at baseline, within \pm 3 days of chemotherapy and/or postintervention (surgical excision). Electronic medical record and/or paper chart review of local control, relapse-free survival and overall survival will occur at 3,5 and 10 years to explore possible effects on long-term outcome. 


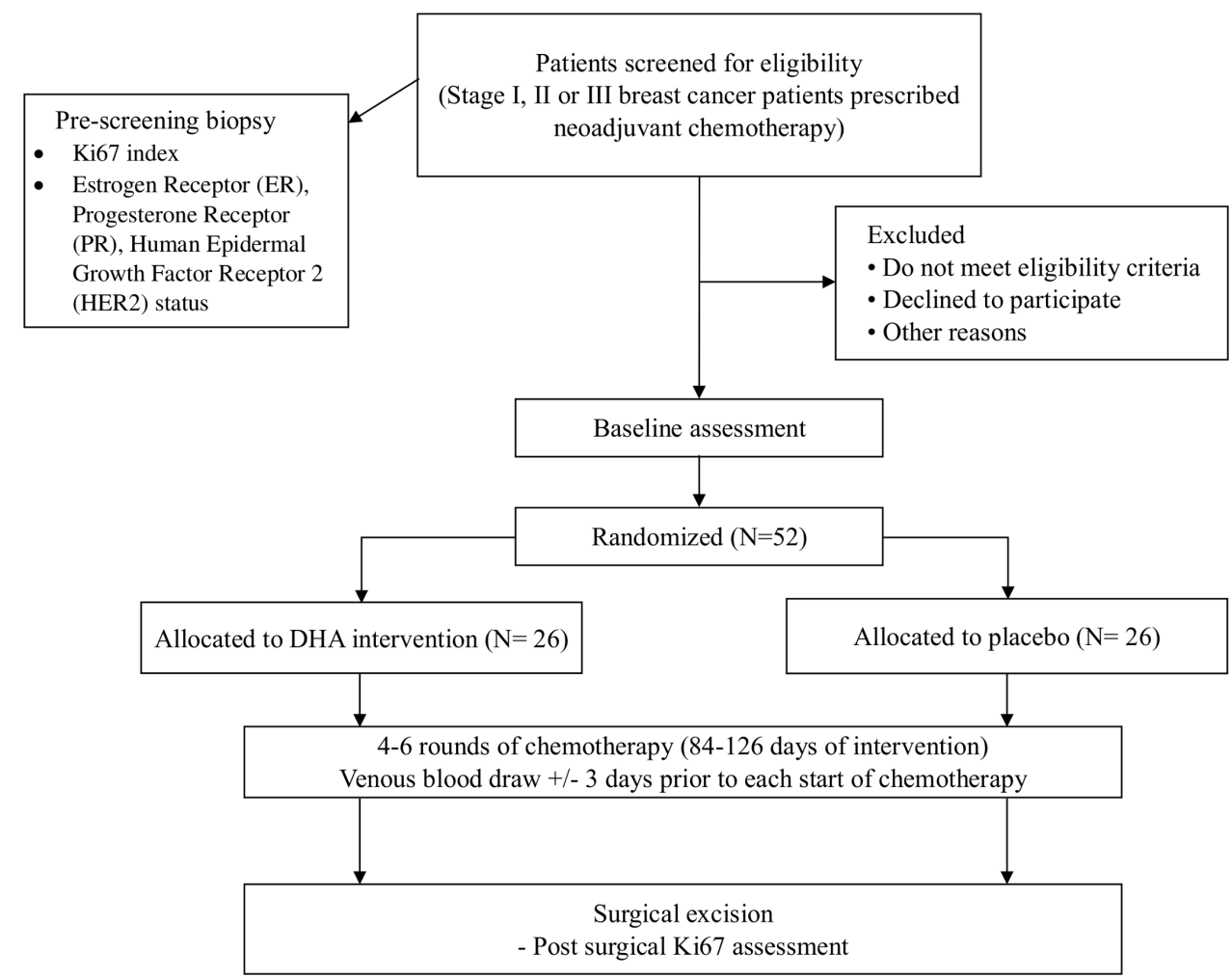

Figure 2 SPIRIT patient flow diagram of the"Docosahexaenoic acid (DHA) for Women with Breast Cancer in the Neoadjuvant Setting" (DHA WIN) trial. DHA, docosahexaenoic acid; SPIRIT, Standard Protocol Items: Recommendations for Interventional Trials.

\section{Primary outcome}

The primary outcome of this study is the change in Ki67 from pretreatment core needle biopsy to surgical excision. It will be calculated by image analysis and will follow the analytical and preanalytical recommendations of Dowsett et al. ${ }^{24}$ The per cent change in Ki67 index at experimental end (surgical excision) from baseline will be determined on a log scale, and the mean per cent change in Ki67 level from baseline will be calculated. Ki67 assays will be performed and reported as part of the routine diagnostic services. A semiautomated computer algorithm scoring system will be employed as previously described ${ }^{36}$ using the platform QuPath. ${ }^{37}$ It is expected that $4.4 \mathrm{~g} \mathrm{DHA} /$ day will result in a clinically relevant decrease in Ki67.

\section{Secondary outcomes}

- DHA incorporation into phospholipids: The changes in the level of DHA incorporation in plasma phospholipids will be assessed at baseline and on day 1 ( \pm 3 days) of each cycle of chemotherapy (2-6) and the end of cycle 6 to identify the range of DHA incorporation in this patient population. The use of plasma rather than red blood cells or whole blood for this study is supported by the recent recommendations for best practices for fatty acids described by Brenna et $a l^{38}$ Analysis of the plasma phospholipid rather than plasma total lipids avoids the postprandial fluctuation of the triacylglycerol pool and is believed to adequately represent the cell membrane composition. ${ }^{38}$ From our hypothesis and previously published data, ${ }^{22}$ it is expected that supplementing with DHA will result in a significant increase in DHA incorporation. If possible, with the small study size, we will also assess differences in DHA incorporation in patients with different breast cancer subtypes and if subtype or disease stage affects DHA incorporation into plasma, controlling for the reported dose taken by the patient. The goal is to determine if plasma phospholipid DHA content can be used to predict treatment outcomes. We will also assess incorporation of other essential fatty acids (linoleic, linolenic, arachidonic, eicosapentaenoic, docosapentaenoic) to determine if there are differences between or within treatment groups.

- Systemic immune function: Systemic immune function will be assessed on blood samples obtained at baseline, beginning of chemotherapy cycle 4 (day $1 \pm 3$ days) and at the end of chemotherapy treatment. Changes in markers of systemic immune cell type and function will be assessed following supplementation compared with baseline and the change from baseline compared with patients receiving the placebo. We will also examine the relationship between changes in activation markers and the level of DHA incorporation, and changes in systemic inflammation ( $\mathrm{C}$ reactive protein (CRP), interleukin (IL)-6, tumour necrosis factor alpha $(\mathrm{TNF} \alpha)$ ) and immune function (ability to produce IL-2 after stimulation in vitro) following DHA supplementation. 


\section{Box 1 Inclusion and exclusion criteria for DHA WIN}

\section{Inclusion criteria.}

1. ECOG performance status of 0 or 1 .

2. Haematology and biochemistry assessments (CBC and differential, partial thromboplastin time, prothrombin time/international normalised ratio, aspartate aminotransferase, alkaline phosphatase, bilirubin and creatinine) within normal range unless determined not clinically significant by the qualified investigator.

3. Ability to take oral medications.

4. Adequate tissue specimen for diagnosis, biomarkers and endpoint Ki67 assays.

\section{Exclusion criteria.}

1. Patients undergoing surgery prior to chemotherapy.

2. Current or previous (within 2 months) daily use ( $>1$ day/week) of omega-3, fish oil, or other supplements or foods containing DHA (at daily doses $>200 \mathrm{mg}$ ).

3. Known allergy to soy or corn.

4. Continued intake of supplements containing vitamin $C$, vitamin $E$ or $\beta$-carotene exceeding the dietary reference intakes (DRI), or other antioxidant supplements.

5. Symptomatic but untreated cholelithiasis.

6. History of deep venous thrombosis, active thrombophlebitis, pulmonary embolism, stroke, acute myocardial infarction, congestive cardiac failure, untreated hypertension and known inherited hypercoagulable disorder.

7. Diagnosis of any other malignancy within the previous year except for adequately treated basal cell or squamous cell skin cancer.

8. Medically documented history of a psychiatric disorder that would preclude consent.

9. Partial or complete loss of vision or diplopia, from ophthalmic vascular disease.

10. Hypersensitivity to any component of the container.

CBC, complete blood count; DHA, docosahexaenoic acid; ECOG, Eastern Cooperative Oncology Group.

- Identify factors that may affect DHA incorporation into plasma phospholipids: If incorporation of DHA into plasma phospholipids is variable within the DHA treatment arm, possible factors that may influence incorporation will be assessed between high and low incorporators. These parameters will be assessed at the end of the study from data collected throughout the study.

- Examine changes in markers for apoptosis: Caspase-3 presence in the excised tumour, as per cent positive cells, will be calculated by image analysis, and a comparison of expression levels at experimental end (surgical excision) and baseline will be determined in patients receiving DHA supplementation and compared with patients receiving placebo. Proportions of negative cells, weakly positive cells and strongly positive cells will be scored by two pathologists, and the staining intensity, assessed by QuPath, ${ }^{37}$ will be recorded independently. Increased apoptosis measured by caspase-3 is a clinically relevant marker of cell death.

- Examine changes in tumour infiltrating lymphocytes (TILs): CD4+ and CD8+ in the excised tumour, as a number of positive cells for a given area, will be calculated by image analysis. A comparison post-treatment of expression levels at experimental end (surgical excision) and baseline will be performed in patients receiving DHA supplementation and compared with patients receiving placebo. The differences will be compared between treatments and within the treatment group, related to plasma DHA concentrations. Increased infiltration of TILs is a potential marker that could be used to predict treatment patient outcomes.

- pCR rate: pCR in resected breast tissue and all sampled axillary nodes will be assessed as absence of invasive cancer by $\mathrm{H} \& \mathrm{E}$ evaluation as per standard of care. pCR will be classified as ypT0/is ypN0 and will be determined at the end of study after surgical resection as part of standard-of-care assessment.

- Comparison of rate of chemotherapy-associated grade 3 and 4 toxicities: The rate of chemotherapy-associated grade 3 and 4 toxicities and chemotherapy-associated hospitalisations will be compared between DHA and placebo arms. Any changes will then be examined in regard to level of supplementation and DHA incorporation. These analyses will be completed at the end of study after surgical resection.

\section{Exploratory outcomes}

- Food Frequency Questionnaire (FFQ): The FFQ will be assessed to compare the estimated (prediagnosis) usual intake of macronutrients on an energy basis (including fat content and composition) between the two groups at baseline. In the future, the overall medians/means of the subjects in this study will be compared with age-matched women in the Alberta's Tomorrow Project.

- Quality of life: Changes in quality of life will be determined by questionnaire employed at baseline and end of treatment. Comparisons will be assessed from end of treatment to baseline within and between treatment groups.

- Exercise behaviour: Exercise behaviour will be determined by questionnaire employed at baseline, each cycle of chemotherapy and end of treatment. Comparisons will be assessed from end of treatment to baseline within and between treatment groups.

- Breast conservation: The rate of breast conservation, specifically the rate of lumpectomy and mastectomy, will be determined by review of surgical and pathological reports at the end of study after surgical resection.

- Volume of surgical blood loss: High intakes of n-3 LCPUFA (that contain some DHA) have been studied to determine if they increase bleeding time. ${ }^{39}{ }^{40} \mathrm{We}$ will review surgical report estimates of blood loss to see if there is a qualitative or quantitative difference between placebo and treatment arms, once adjusted for the magnitude of surgery (lumpectomy vs mastectomy vs mastectomy + immediate reconstruction; sentinel node dissection vs full axillary dissection). It is not expected that we will see a difference as it is an 


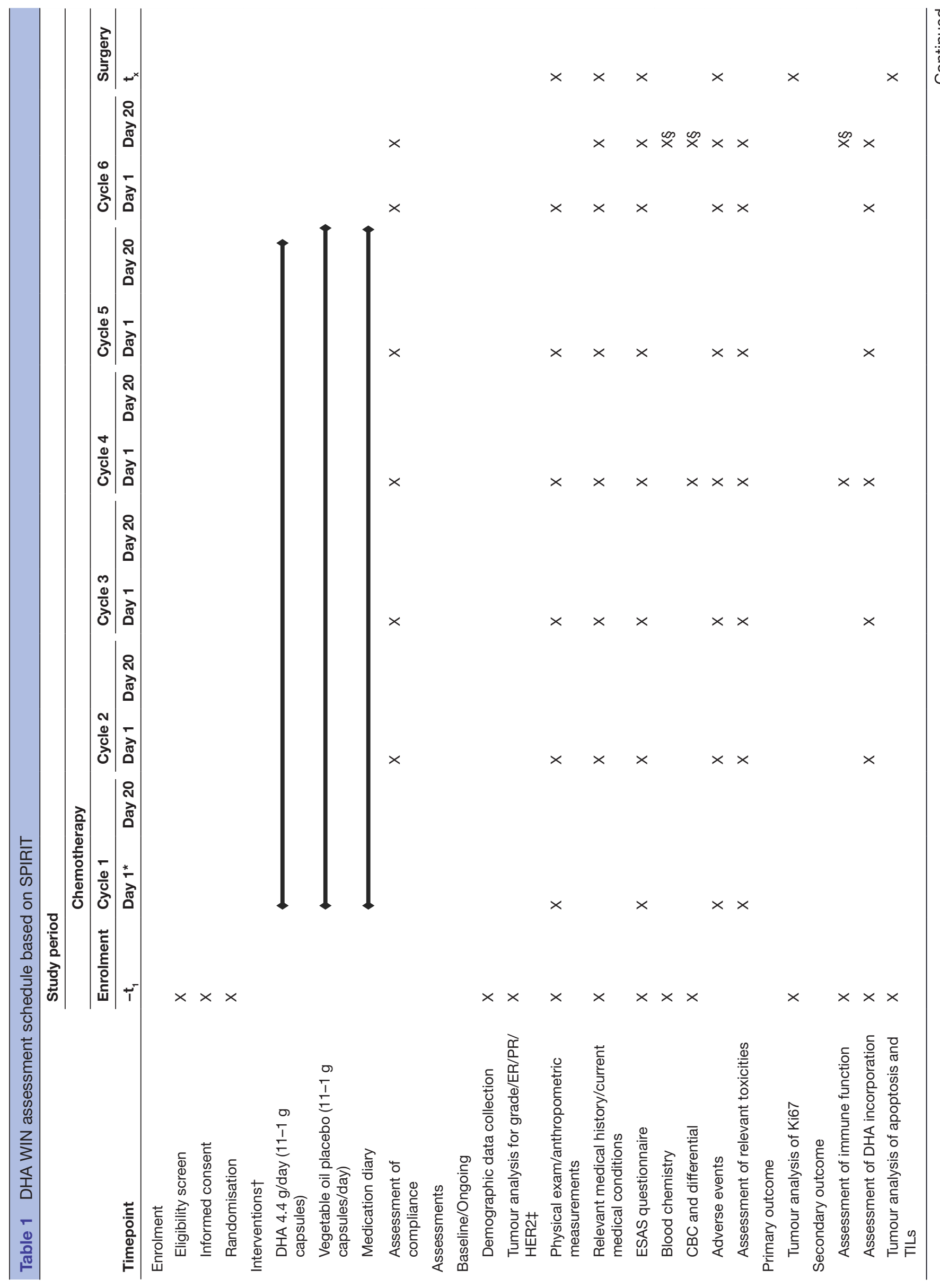

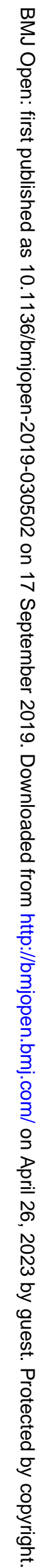




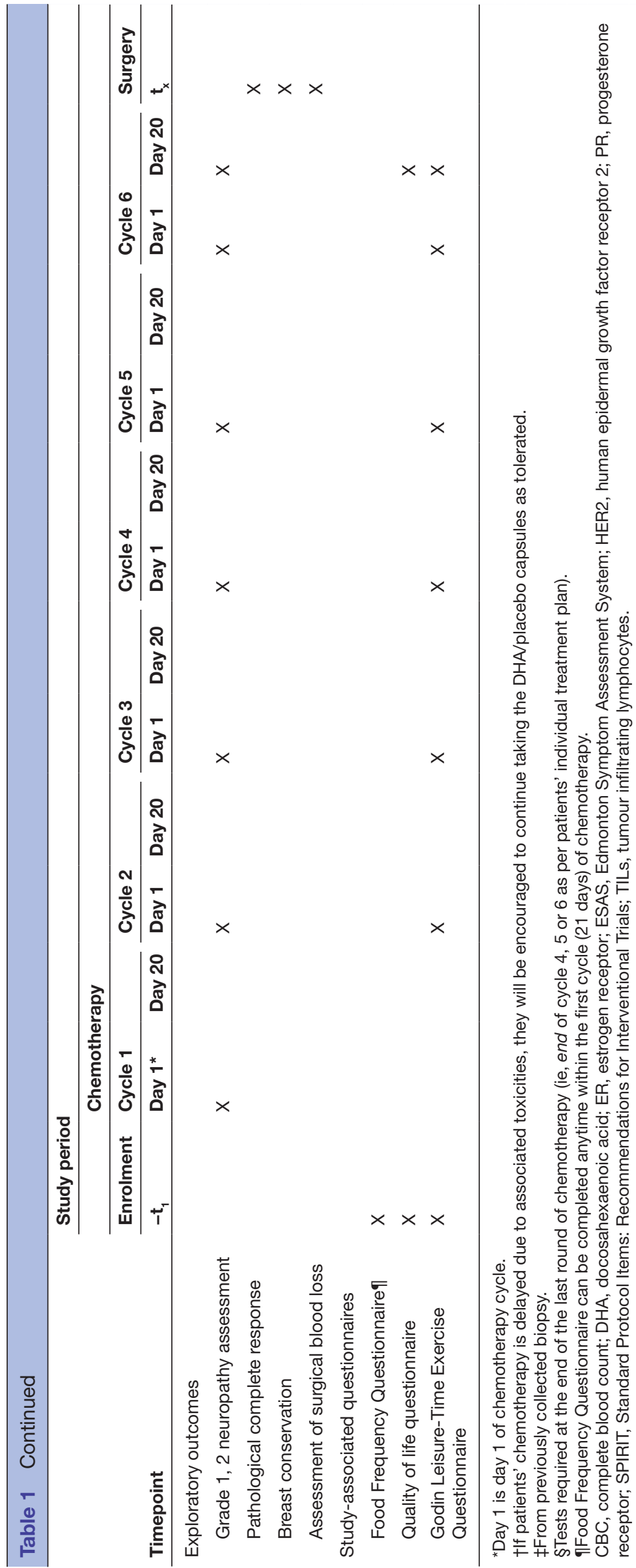


eicosapentaenoic acid (the precursor to DHA), which has antithrombotic and antiplatelet properties. ${ }^{41}$

- Local control, relapse-free survival and overall survival: Local control, relapse-free survival and overall survival will be analysed by review of electronic medical records, registry reports and/or paper medical charts at 3,5 and 10 years to explore possible effects on longterm outcome.

\section{Participant timeline}

Patients with breast cancer receiving neoadjuvant chemotherapy account for approximately $20 \%$ of newly diagnosed patients with breast cancer, approximately 10-12 per month at the Cross Cancer Institute. Assuming a conservative accrual rate of $30 \%$, accrual is estimated to be completed in 14-18 months with 3-4 patients recruited per month. Each patient will be enrolled for the duration of their individual chemotherapy regimen, an estimated 12-18 weeks (84-126 days) beginning at the start of the first cycle of chemotherapy and continued through 4-6 cycles of chemotherapy (3 weeks/cycle). The intervention will be discontinued 21-35 days after the last administration of cytotoxic chemotherapy when surgery to remove the tumour occurs. See figure 1 for a schematic of the participant timeline.

\section{Sample size}

Fifty-two women prescribed neoadjuvant breast cancer chemotherapy will be enrolled in a two-arm trial with 26 participants per arm. The sample size calculation is based on the primary objective, which is to determine the efficacy of supplemental DHA provided with standard neoadjuvant as measured by change in the Ki67 index from biopsy to surgical excision. Group sample sizes of 23 patients in each group are required to achieve $81 \%$ power to detect a difference between the group proportions of 0.4 . The proportion in group 1 is assumed to be 0.3 under the null hypothesis and 0.7 under the alternate hypothesis. The proportion in group 2 , which is the control group, is 0.3 . The test statistic used is the two-sided t-test. The significance level of the test was targeted at 0.05 , and the significance level actually achieved by this design is about 0.0497 . Assuming a dropout rate estimated at approximately $10 \%$ for this patient population, which is approximately 5 patients, a total of 52 patients (26 patients in the DHA supplementation group and 26 in the placebo group) are required for the study.

\section{Recruitment}

Oncologists and clinical trial nurses at the Cross Cancer Institute in Edmonton, Canada, will recruit newly diagnosed patients with breast cancer. Patients will be screened for eligibility by the clinical trial nurses, and eligible, interested patients will receive a detailed explanation of the study by the study coordinators and written informed consent will be obtained (online supplementary file 1).

\section{Randomisation and blinding}

A biostatistician will generate a patient randomisation list and randomised bottle numbers by covariate-adaptive randomisation (block randomisation). The randomised bottle numbers will be provided to DSM for labelling for both the DHA and placebo groups, and the randomised bottle list will also be provided to the unblinded Clinical Trials Coordinator (CTC, Clinical Trials Unit) and the unblinded pharmacist. Patients will be stratified by histological subtype and then randomised. The allocation of the study arm (as the study is blinded, study arms A and B will be used as this will not identify the placebo or intervention arm) and a unique study identifier will be conducted using the REDCap database. The key to study arms A and B will only be provided to the unblinded CTC, statistician and the pharmacist. The study coordinator will enter the new patient information in REDCap and assign the unique identification (ID) and arm. This information will be shared with the unblinded CTC and the unblinded pharmacy staff. The pharmacy staff will assign the correct bottle numbers based on the study arm on day 1 of each chemotherapy cycle. Following the allocation of the bottle numbers, this information will then be shared with the study coordinator and the unblinded CTC. The bottle ID will be entered in the REDCap database by the study coordinator. All future bottle allocations with the unique bottle ID will be entered into the REDCap database. The key to the study arm will be kept in password-protected computers and will only be shared in an urgent need for breaking of the blind. When a blinding code is broken, the date and reason for unblinding must be fully documented in source documents and entered on the case report form. Every effort should be made by site staff to ensure that the treatment arm in which the unblinded patient is assigned is communicated only to those site staff that require the information for treatment purposes. To assist in maintaining the blind of the patients, supplements and placebo are identical in size, shape, colour and texture, in addition to identical bottles for dispensing. Patients, pathologists, physicians and researchers will be blinded to patient enrolment in the study and throughout the trial. Blinding will only be dropped after analysis of fatty acids, systemic immune function and Ki67 is complete.

\section{Data collection, management and analysis}

Study methods are summarised in table 2. Briefly, data will be collected and measured at baseline, within \pm 3 days of chemotherapy and/or postintervention (surgical excision). Electronic medical record and/or paper chart review of local control, relapse-free survival and overall survival will occur at 3,5 and 10 years. All data will be entered and maintained in the REDCap trial database. Baseline measurements will be analysed once all participants have been enrolled, and all other analyses will occur at completion of trial. 
Table 2 Variables, measures and methods of analysis

\begin{tabular}{|c|c|c|c|}
\hline Variable/Outcome & Outcome measure & Method & Statistical analysis \\
\hline \multicolumn{4}{|l|}{ Primary } \\
\hline $\begin{array}{l}\text { Efficacy of supplemental } \\
\text { DHA provided with standard } \\
\text { neoadjuvant chemotherapy } \\
\text { as measured by change in } \\
\text { Ki67. }\end{array}$ & Ki67 labelling index. & Immunohistochemistry. & $\begin{array}{l}95 \% \mathrm{t}-\mathrm{Cl} \text { for mean per cent change } \\
\text { in } \mathrm{Ki} 67 . \\
\text { Independent t-test to compare } \\
\text { change between the study groups. }\end{array}$ \\
\hline
\end{tabular}

\section{Secondary}

1. DHA incorporation into Fatty acid composition of plasma plasma phospholipids.

\section{phospholipids.}

Gas chromatography.

Paired t-test will be used to compare the mean per cent change in the DHA level of patients after each cycle with their baseline values. If the data are not normally distributed, the Wilcoxon signed-rank test will be employed for this comparison. A 95\% $\mathrm{t}-\mathrm{Cl}$ for the mean per cent change in the DHA from baseline will be compared with patients receiving placebo.

$\begin{array}{llll}\text { 2. Systemic immune } & \text { Immune cell subset identification. } & \text { Flow cytometry. } & \text { Repeated-measures analysis of } \\ \text { function. } & \text { Plasma cytokines. } & \text { ELISA. } & \text { variance with post-hoc analysis. } \\ & \text { Eesponse. } & \text { Meso Scale. } & \end{array}$

3. Identify factors that may Factors assessed after calculating high affect DHA incorporation and low DHA incorporators:

into tumour tissue and Weight (body mass index-BMI).

plasma phospholipids. Age.

- Usual diet estimated from the FFQ.

- Composition of dietary fat estimated from the FFQ.

- Histology of the tumour (provided from the biopsy).

- Amount of DHA consumed (adherence to the supplement).

\% incorporation of other fatty acids.
4. Examine changes in
Caspase-3.
Immunohistochemistry. markers for apoptosis.
CD4+/CD8+. Immunohistochemistry. 5. Examine changes
in markers for tumour
infiltrating lymphocytes.
6. Describe the rate of pCR in breast and in axillary nodes.
Absence of invasive cancer on $\mathrm{H} \& \mathrm{E}$ evaluation.
Immunohistochemistry.
$\mathrm{pCR}=\mathrm{ypTO} /$ is ypNO.
95\% $\mathrm{t}-\mathrm{Cl}$ using independent t-test for mean per cent change between treatment groups.

\begin{tabular}{|c|c|c|c|}
\hline $\begin{array}{l}\text { 7. Describe the rate } \\
\text { of grade } 3 \text { and } 4 \\
\text { chemotherapy-associated }\end{array}$ & $\begin{array}{l}\text { Rate of grade } 3 / 4 \text { toxicities } \\
\text { and chemotherapy-associated } \\
\text { hospitalisations. }\end{array}$ & Chart review. & $\begin{array}{l}95 \% \mathrm{t}-\mathrm{Cl} \text { using independent } \mathrm{t} \text {-test } \\
\text { for mean per cent change in events } \\
\text { between treatment groups. }\end{array}$ \\
\hline
\end{tabular}

Independent t-test will be conducted to compare the mean values between the two study groups. $\chi^{2}$ test will be conducted to determine correlation between two categorical variables for outcome measures listed. toxicities.

\begin{tabular}{lll}
\hline $\begin{array}{l}\text { Exploratory outcomes } \\
\text { 1. FFQ. }\end{array}$ & Diet History Questionnaire II & $\begin{array}{l}\text { Questionnaire. } \\
\text { Independent t-test of macronutrient } \\
\text { and fat content/composition between } \\
\text { groups. }\end{array}$ \\
\hline 2. Quality of life. & Baseline and endpoint questionnaires. & $\begin{array}{l}\text { Paired t-test for continuous variables } \\
\text { and McNemar's for categorical } \\
\text { variables for mean per cent change } \\
\text { in events between treatment groups. }\end{array}$ \\
\hline
\end{tabular}


Table 2 Continued

\begin{tabular}{|c|c|c|c|}
\hline Variable/Outcome & Outcome measure & Method & Statistical analysis \\
\hline 3. Exercise. & $\begin{array}{l}\text { Godin Leisure-Time Exercise } \\
\text { Questionnaire. }\end{array}$ & Questionnaire. & $\begin{array}{l}\text { Paired t-test for continuous variables } \\
\text { and McNemar's for categorical } \\
\text { variables for mean per cent change } \\
\text { in events between treatment groups. }\end{array}$ \\
\hline $\begin{array}{l}\text { 4. Assess the rate of } \\
\text { breast conservation. }\end{array}$ & Rate of lumpectomy and mastectomy. & Chart review. & $\chi^{2}$ tests. \\
\hline $\begin{array}{l}\text { 6. Analyse local control, } \\
\text { relapse-free survival and } \\
\text { overall survival. }\end{array}$ & $\begin{array}{l}\text { Electronic medical record and/or paper } \\
\text { medical chart review at } 3,5 \text { and } 10 \\
\text { years to explore possible effects on } \\
\text { long-term outcome. }\end{array}$ & Chart review. & $\begin{array}{l}\text { Kaplan-Meier estimates along with } \\
\text { the survival curves, log-rank test will } \\
\text { be used for statistical comparison } \\
\text { between groups. }\end{array}$ \\
\hline
\end{tabular}

DHA, docosahexaenoic acid; FFQ, Food Frequency Questionnaire; GEE, generalised estimating equation; pCR, pathological complete response.

\section{Primary outcome}

Ki67 will be tested by IHC by the diagnostic biomarker laboratory at the Cross Cancer Institute using the MIB1 antibody on $4 \mu \mathrm{m}$ sections from formalin fixed paraffin embedded (FFPE) needle core biopsy surgical specimens. At final analyses, Ki67 staining will be repeated as a single IHC stain and interpreted by image analysis. At the time of Ki67 interpretation, slides will be de-identified and coded to ensure the pathologist is blinded to the experimental group. In addition, the original single-stained slides will be interpreted visually by research staff. All Ki67 values (routine and image analysis) will be recorded as absolute percentage and H-score in the REDCap trial database and the participants' case report form. The Ki67 index is validated and used in clinic as a marker of proliferation. The Ki67 index (absolute \% and H-score ${ }^{42}$ ) of biopsy and surgical resection (after chemotherapy) will be compared on each participant and between participants receiving DHA compared with placebo.

\section{Secondary outcomes}

DHA incorporation into plasma phospholipids will be measured in venous blood from patients at baseline (time of enrolment in trial) and on day 1 ( \pm 3 days) of each chemotherapy cycle by a technician blinded to the treatment group. Venous blood will be collected in coated EDTA tubes and centrifuged at $750 \times g$ for $10 \mathrm{~min}$ to obtain plasma. Red blood cells will be immediately frozen and banked at $-80^{\circ} \mathrm{C}$ for storage for future secondary analysis. Plasma will be separated into six aliquots and immediately frozen at $-80^{\circ} \mathrm{C}$ for storage. Plasma will be extracted by the Folch procedure, ${ }^{43} 44$ phospholipids separated by thin layer chromatography and fatty acid content (concentration and relative per cent) measured by gas-liquid chromatography as previously described. ${ }^{45}$ The percentage change in DHA from baseline will be compared in each patient and a $95 \%$ t-CI for the mean per cent change in DHA from baseline will be compared with patients receiving placebo. An internal standard is used to identify and quantify the fatty acids. This standard measure for fatty acid status has coefficient of variation $<5 \%$, and individual gas chromotography (GC) peaks are validated against phospholipid standards (GLC-502 and GLC-643) from NuChek (Elysian, Minnesota).

Phenotyping of immune cell subsets will be measured using whole blood (collected in EDTA tubes). The various cell types will be identified using specific fluorescently labelled monoclonal antibodies to surface receptors (see online supplementary table 4 for list of antibodies). These will be quantified by flow cytometry, as previously described. ${ }^{46}$ With the remaining blood, peripheral mononuclear cells will be isolated and purified on a Ficoll density gradient of Histopaque-1077 as previously described. ${ }^{46}{ }^{47}$ To measure cytokine production in isolated lymphocytes, cells will be cultured in media with or without the mitogens, phytohemagglutinin or lipopolysaccharide, for 48 hours as previously described. ${ }^{48}$ Supernatant will be collected and stored at $-80^{\circ} \mathrm{C}$ for ex vivo measures of immune function (ability and pattern of cytokines produced after stimulation). IL-1 $\beta$, IL-2, IL-6, IL-10, TNF $\alpha$ and interferon- $\gamma$ (pg/ $\mathrm{mL}$ ) cytokines will be measured using electrochemiluminescent multiplex assays (Meso Scale Discovery) or by individual ELISA assays. Cytokines listed above and inflammatory markers including CRP in plasma will be measured by electrochemiluminescent multiplex assays (Meso Scale Discovery) as previously described. ${ }^{49}$ Cytokines and inflammatory markers in plasma and cytokines from cultured lymphocytes will be analysed when all samples have been collected. Changes in systemic immune function will be assessed in patients compared with baseline and compared between groups. The data analysis will occur at completion of trial. Cytokines are done in duplicate and the coefficient of variance is $<15 \%$. Phenotypes will be collected as a relative per cent of total cells and the change compared between treatments. Additionally, white blood cells that are not used for the immune assays will be assessed for fatty acid composition. 
If DHA incorporation into plasma phospholipids is significantly different within the DHA supplementation arm, factors that may influence incorporation will be compared in low versus high incorporators, to identify possible factors that predict incorporation, including body mass index (BMI), age, the estimated macronutrient intake and composition of dietary fat of women (estimated from the FFQ), histology of the tumour (provided from the biopsy), the amount of DHA consumed (adherence to the supplement) and length of time DHA was consumed (if treatment is ended early). We will also assess incorporation of other fatty acids (palmitic, oleic, linoleic, linolenic, arachidonic, eicosapentaenoic, docosapentaenoic) to determine if there are differences between or within treatment groups.

Caspase-3 changes and changes in CD4 and CD8 will be tested by IHC by the diagnostic biomarker laboratory at the Cross Cancer Institute on $4 \mu \mathrm{m}$ sections from FFPE surgical specimens. At final analyses, IHC staining will be interpreted by image analysis. At time of interpretation, slides will be de-identified and coded to maintain the blind. All values (routine and image analysis) will be recorded as absolute percentage. Caspase-3 is a validated marker of apoptosis, and CD4 and CD8 are validated markers for lymphocytes. The calculated indices (absolute $\%$ and $\mathrm{H}$-score) of biopsy and surgical resection will be compared on each participant and between participants receiving DHA compared with placebo.

pCR in resected breast tissue and axillary nodes will be assessed in H\&E-stained tissue for evidence of invasive disease according to standard of care and recorded in patients' case report form. The rate of pCR in breast tissue and axillary nodes after surgical resection will be compared between participants receiving DHA supplementation compared with placebo.

Grade 3 and 4 toxicities will be assessed and recorded by the clinical trial nurse. Toxicities will be assessed on day 1 ( \pm 3 days) of each chemotherapy cycle. Dates of hospitalisation will be recorded in patients' case report form. Rates of chemotherapy-associated grade 3/4 toxicities, all-grade neuropathy and hospitalisations will be compared between DHA supplementation and placebo arms as scored by a medical oncologist in a standardised toxicity/neuropathy form for each cycle of chemotherapy.

\section{Exploratory outcomes}

The FFQ is a validated questionnaire for macronutrient intake. ${ }^{50-52}$ The quality of life questionnaire is a validated questionnaire from the European Organisation for Research and Treatment of Cancer-Quality of Life Questionnaire-C30. ${ }^{53}$ Exercise behaviour will be assessed using the modified Godin Leisure-Time Exercise Questionnaire ${ }^{5455}$ Assessment of changes in quality of life and exercise behaviour will be compared from timepoints collected to baseline within and between treatment groups. We do not expect the supplement/placebo to influence this variable, but since exercise alters immune function, quality of life and tumour growth we have included it herein to determine if it changes during therapy.

The rate of breast conservation, specifically the rate of lumpectomy and modified radical mastectomy, will be determined by surgical and pathological reports at time of surgical resection. Volume estimates of blood loss will be assessed by review of surgical reports to see if there is a qualitative or quantitative difference between placebo and treatment arms, once adjusted for the type of surgery (lumpectomy vs mastectomy vs mastectomy + immediate reconstruction; sentinel node dissection vs full axillary dissection). Local control, relapse-free survival and overall survival will be analysed by electronic medical record and/or paper medical chart review at 3,5 and 10 years. Data will be validated by a medical oncologist.

\section{Data management}

All data will be entered and maintained in the REDCap trial database. Direct access to clinical and laboratory information on enrolled trial patients will be limited to the principal investigator, coinvestigators, trainees/staff who have had the appropriate training and approval, and study nurses and study coordinators who will have access to the source documents through the electronic medical record and laboratory information system at the Cross Cancer Institute. All patients will have biopsy and tumour samples for analysis, and we do not expect any missing data for the primary endpoint (Ki67). If supplement compliance is below $50 \%$ for more than two consecutive cycles, or if participants do not complete chemotherapy (to a minimum of four cycles), they will be excluded from final analysis of the primary endpoint. If patients do not have sufficient blood samples for the secondary analyses (DHA incorporation, systemic immune function), analysis will be performed using data from the remaining patients.

\section{Statistical methods \\ Primary outcome}

The per cent change in Ki67 will be determined as an absolute percentage and $\mathrm{H}$-score. The number of patients showing a decrease and the 95\% CI for the mean per cent change in the Ki67 level from baseline in patients receiving DHA supplementation will be compared with patients receiving placebo. The mean change will be measured using an independent t-test between the two groups.

\section{Secondary outcomes}

Paired t-tests will be used to compare the mean per cent change in the plasma DHA level of the patients after each cycle of chemotherapy with their baseline values. If the data are not normally distributed, the Wilcoxon signedrank test will be used to compare the plasma DHA level after each cycle of chemotherapy with baseline. The difference in plasma phospholipid DHA from baseline and between DHA supplementation and placebo arms will be calculated, and the $95 \%$ CI for the mean per cent 
change in DHA level from baseline and groups will be assessed.

If systemic immune function data are not normally distributed, it will be log-transformed prior to analysis and the normality assumptions will be tested again. Repeated-measures analysis of variance with post-hoc analysis will be used to determine if there is an effect of treatment on immune function.

Factors affecting DHA incorporation will be examined by independent t-tests to compare the mean values between the DHA and placebo groups. $\chi^{2}$ tests will be conducted to determine correlation between two categorical variables for the outcome measures listed.

The within-subject and between-subject variability between the two groups for the mean per cent change in apoptosis and TIL markers will be tested using the generalised estimating equation method.

The 95\% CI using independent t-tests will be conducted for the mean per cent change in pCR and rates of grade 3 and 4 chemotherapy-associated toxicities and hospitalisation in patients receiving DHA supplementation compared with patients receiving placebo.

\section{Exploratory outcomes}

Independent t-tests for macronutrient and fat content obtained from the FFQ will be examined between groups. Paired t-tests for continuous variables and McNemar's test for categorical variables will be assessed for mean per cent change in events between treatment arms for the quality of life and exercise questionnaires. $\chi^{2}$ tests will be used to compare the degree of breast conservation, and the volume of surgical blood loss will employ an independent t-test between the two study arms. Rate of local control will be compared between treatment arms using t-test of proportions. Recurrence-free survival and survival will be analysed using the log-rank test on Kaplan-Meier survival curves.

SAS V.9.4 software will be used for statistical analysis. A $p$ value $<0.05$ will be used for statistical significance. Two-sided tests will be used for all statistical tests.

\section{Data monitoring}

The trial activities performed at the Cross Cancer Institute will be monitored by the Cross Cancer Institute, Investigator Initiated Trials Data Safety Monitoring Board (DSMB). The DSMB is independent of the investigator and is composed of representatives from both medical and radiation oncology.

The investigator will assess the relationship between protocol treatment and the occurrence of adverse events, and this assessment will be recorded in the database for adverse events. This study will use the International Common Terminology Criteria for Adverse Events V.5.0 for adverse event reporting. The reporting period for adverse events will start at the time the patient takes the first dose of DHA/placebo through and including 28 calendar days after last administration of study agent. If serious adverse reaction to treatment occurs, the Natural and Non-prescription Health Products Directorate, Clinical Trials Unit, Health Research Ethics Board of Alberta - Cancer Committee (HREBA.CC) and DSM will be notified as per guidelines. After 25 evaluable patients, all data and results will be submitted to the DSMB for review.

\section{Auditing}

As per the SPIRIT guidelines, the investigators, Cross Cancer Institute and the University of Alberta will permit trial-related monitoring, audits, Research Ethics Board, DSMB review and regulatory inspection(s), providing direct access to paper and/or electronic documentation pertaining to the clinical study (eg, case report forms, source documents such as hospital patient charts and investigator study files). All site facilities related to the study conduct could be visited during an audit (eg, pharmacy, laboratory, outpatient department) and are agreed to cooperate and provide assistance at reasonable times and places with respect to any auditing activity.

\section{Patient and public involvement}

Patients were not involved in the protocol development or study design. However, oncologists and clinical trial nurses who work in the breast tumour group are involved in patient screening to assess eligibility for the study. The HREBA.CC-approved informed consent will be obtained from patients prior to their involvement in the study, and it informs patients of their right to withdraw at any time. At the end of the trial, results will be disseminated to the public through seminars, public talks and in peer-reviewed journals.

\section{ETHICS AND DISSEMINATION}

The study is registered at ClinicalTrials.gov. Protocol amendments will be submitted to HREBA.CC, Health Canada and the clinical trial registry prior to study implementation according to regulatory requirements. The formal consent of a participant, using the HREBA.CC-approved consent form (online supplementary file 1), will be obtained by a clinical trial nurse before the participant is enrolled in the study and will be signed by the patient and the principal investigator. A voluntary optional consent form for use of participant data and biological specimens (online supplementary file 2) will be offered at time of enrolment. Patient confidentiality and anonymity will be maintained and identities protected from unauthorised parties.

Access to data will be restricted to the primary investigators and statistician. They will grant access to other team members as governed and approved by ethics. Ancillary care post-trial will occur as routine standard of care for all participants. Our objective is to determine the efficacy of using DHA supplementation concomitant with chemotherapy, and as such our results will be disseminated to clinicians for implementation in future treatment paradigms. The results will be submitted to peer-reviewed 
journals and presented at national and international conferences.

\section{Author affiliations}

${ }^{1}$ Department of Agricultural, Food and Nutritional Science, University of Alberta, Edmonton, Alberta, Canada

${ }^{2}$ Department of Oncology, University of Alberta, Edmonton, Alberta, Canada

${ }^{3}$ Alberta Health Services, Edmonton, Alberta, Canada

${ }^{4}$ Department of Laboratory Medicine and Pathology, University of Alberta, Edmonton, Alberta, Canada

${ }^{5}$ Faculty of Kinesiology, Sport and Recreation, University of Alberta, Edmonton, Alberta, Canada

Contributors MN and CF wrote the manuscript. JRM, GB, MA-C, SGo, SGh, AS, DM, AC, LP, KB, VM, KC, RB, W-FD, GW, SKB, AAJ, KK, JM-J and XZ contributed to the study design and reviewed the manuscript drafts. JRM, SKB, AAJ, KK, JM-J and XZ provided oncological expertise. GB, RB, W-FD and GW provided pathology and immunohistochemistry expertise. SGo and KB contributed to the design of the immunological component of the study. SGh designed the statistical models for all components of the study. AS, DM, AC and MA-C obtained all regulatory and operational approvals (Health Canada, HREBA, clinical trials registration and site approvals). CF, LP, VM, KB and JRM were coapplicants on the successful CIHR grant that designed the immune component of the trial. KC contributed expertise for the $\mathrm{Q} \mathrm{L}$ and exercise component of the study. All authors reviewed the drafts of the manuscript. Authors of the data manuscripts will include at least the principal investigator, medical director (JRM) and any coinvestigators who have (1) included eligible patients in the trial (by order of inclusion) and/or (2) contributed significantly to the design, conduct and data interpretation regarding companion basic science studies.

Funding This study is supported by the Canadian Institutes of Health Research (grant number: RES0037745), Cross Cancer Institute Investigator Initiated Trials (grant number: IIT-0005) and a gift from the Butler Family Foundation, Edmonton, Alberta.

\section{Competing interests None declared.}

Patient consent for publication Not required.

Ethics approval DHA WIN has received Health Canada approval (\#HC6-24-c220167) and full ethical approval from the Health Research Ethics Board of Alberta - Cancer Committee (HREBA.CC-18-0381).

Provenance and peer review Not commissioned; externally peer reviewed.

Open access This is an open access article distributed in accordance with the Creative Commons Attribution Non Commercial (CC BY-NC 4.0) license, which permits others to distribute, remix, adapt, build upon this work non-commercially, and license their derivative works on different terms, provided the original work is properly cited, appropriate credit is given, any changes made indicated, and the use is non-commercial. See: http://creativecommons.org/licenses/by-nc/4.0/.

\section{REFERENCES}

1. World Health Organization. Cancer. Available: http://www.who.int/ mediacentre/factsheets/fs297/en/

2. Mamounas EP, Fisher B. Preoperative (neoadjuvant) chemotherapy in patients with breast cancer. Semin Oncol 2001;28:389-99.

3. Teshome M, Hunt KK. Neoadjuvant therapy in the treatment of breast cancer. Surg Oncol Clin N Am 2014;23:505-23.

4. Burdge GC, Wootton SA. Conversion of alpha-linolenic acid to palmitic, palmitoleic, stearic and oleic acids in men and women. Prostaglandins Leukot Essent Fatty Acids 2003;69:283-90.

5. Calder PC. Docosahexaenoic acid. Ann Nutr Metab 2016;69:8-21.

6. Plourde M, Chouinard-Watkins R, Vandal M, et al. Plasma incorporation, apparent retroconversion and $\beta$-oxidation of 13C-docosahexaenoic acid in the elderly. Nutr Metab 2011;8.

7. Chapkin RS, McMurray DN, Davidson LA, et al. Bioactive dietary long-chain fatty acids: emerging mechanisms of action. Br J Nutr 2008;100:1152-7.

8. Schley PD, Brindley DN, Field CJ. (n-3) PUFA alter raft lipid composition and decrease epidermal growth factor receptor levels in lipid rafts of human breast cancer cells. J Nutr 2007;137:548-53.

9. Rogers KR, Kikawa KD, Mouradian M, et al. Docosahexaenoic acid alters epidermal growth factor receptor-related signaling by disrupting its lipid raft association. Carcinogenesis 2010;31:1523-30.
10. Lee EJ, Yun U-J, Koo KH, et al. Down-Regulation of lipid raftassociated onco-proteins via cholesterol-dependent lipid raft internalization in docosahexaenoic acid-induced apoptosis. Biochim Biophys Acta 2014;1841:190-203.

11. Ewaschuk JB, Newell M, Field CJ. Docosahexanoic acid improves chemotherapy efficacy by inducing CD95 translocation to lipid rafts in ER(-) breast cancer cells. Lipids 2012;47:1019-30.

12. Newell M, Brun M, Field CJ. Treatment with DHA modifies the response of MDA-MB-231 breast cancer cells and tumors from nu/ nu mice to doxorubicin through apoptosis and cell cycle arrest. $J$ Nutr 2019;149:46-56.

13. Kang KS, Wang $P$, Yamabe N, et al. Docosahexaenoic acid induces apoptosis in MCF-7 cells in vitro and in vivo via reactive oxygen species formation and caspase 8 activation. PLoS One 2010;5:e10296.

14. Schley PD, Jijon HB, Robinson LE, et al. Mechanisms of omega-3 fatty acid-induced growth inhibition in MDA-MB-231 human breast cancer cells. Breast Cancer Res Treat 2005;92:187-95.

15. Ghosh-Choudhury T, Mandal CC, Woodruff K, et al. Fish oil targets PTEN to regulate NFkappaB for downregulation of antiapoptotic genes in breast tumor growth. Breast Cancer Res Treat 2009;118:213-28.

16. Manni A, Richie JP, Xu H, et al. Influence of omega-3 fatty acids on tamoxifen-induced suppression of rat mammary carcinogenesis. Int J Cancer 2014;134:1549-57.

17. Mason JK, Klaire S, Kharotia S, et al. $\alpha$-linolenic acid and docosahexaenoic acid, alone and combined with trastuzumab, reduce HER2-overexpressing breast cancer cell growth but differentially regulate HER2 signaling pathways. Lipids Health Dis 2015;14:91.

18. Chauvin L, Goupille C, Blanc C, et al. Long chain n-3 polyunsaturated fatty acids increase the efficacy of docetaxel in mammary cancer cells by downregulating Akt and $\mathrm{PKC} \varepsilon / \delta$-induced ERK pathways. Biochim Biophys Acta 2016;1861:380-90.

19. Barascu A, Besson P, Le Floch O, et al. Cdk1-Cyclin B1 mediates the inhibition of proliferation induced by omega-3 fatty acids in MDA-MB-231 breast cancer cells. Int J Biochem Cell Biol 2006;38:196-208.

20. Yee LD, Lester JL, Cole RM, et al. Omega-3 fatty acid supplements in women at high risk of breast cancer have dose-dependent effects on breast adipose tissue fatty acid composition. Am J Clin Nutr 2010;91:1185-94.

21. Bougnoux $P$, Germain $E$, Chajès $V$, et al. Cytotoxic drugs efficacy correlates with adipose tissue docosahexaenoic acid level in locally advanced breast carcinoma. Br J Cancer 1999;79:1765-9.

22. Bougnoux P, Hajjaji N, Ferrasson MN, et al. Improving outcome of chemotherapy of metastatic breast cancer by docosahexaenoic acid: a phase II trial. Br J Cancer 2009;101:1978-85.

23. Morland SL, Martins KJB, Mazurak VC. N-3 polyunsaturated fatty acid supplementation during cancer chemotherapy. J Nutr Intermed Metab 2016:5:107-16.

24. Dowsett M, Nielsen TO, A'Hern R, et al. Assessment of Ki67 in breast cancer: recommendations from the International Ki67 in breast cancer Working group. J Natl Cancer Inst 2011;103:1656-64.

25. Gerdes J, Lemke H, Baisch $\mathrm{H}$, et al. Cell cycle analysis of a cell proliferation-associated human nuclear antigen defined by the monoclonal antibody Ki-67. J Immunol 1984;133:1710-5.

26. Scholzen T, Gerdes J, Thomas S. The Ki-67 protein: from the known and the unknown. J Cell Physiol 2000;182:311-22.

27. Jones RL, Salter J, A'Hern R, et al. The prognostic significance of Ki67 before and after neoadjuvant chemotherapy in breast cancer. Breast Cancer Res Treat 2009;116:53-68.

28. Matsubara N, Mukai H, Fujii S, et al. Different prognostic significance of Ki-67 change between pre- and post-neoadjuvant chemotherapy in various subtypes of breast cancer. Breast Cancer Res Treat 2013;137:203-12.

29. Chan A-W, Tetzlaff JM, Altman DG, et al. Spirit 2013 statement: defining standard protocol items for clinical trials. Ann Intern Med 2013:158:200-7.

30. Chan A-W, Tetzlaff JM, Gøtzsche PC, et al. Spirit 2013 explanation and elaboration: guidance for protocols of clinical trials. BMJ 2013;346:e7586.

31. Arnaout A, Lee J, Gelmon K, et al. Neoadjuvant therapy for breast cancer: updates and proceedings from the seventh annual meeting of the Canadian Consortium for locally advanced breast cancer. Curr Oncol 2018;25:e490-8

32. Roché $H$, Fumoleau $P$, Spielmann $M$, et al. Sequential adjuvant epirubicin-based and docetaxel chemotherapy for node-positive breast cancer patients: the FNCLCC PACS 01 trial. J Clin Oncol 2006;24:5664-71. 
33. Slamon D, Eiermann W, Robert N, et al. Adjuvant trastuzumab in HER2-positive breast cancer. N Engl J Med 2011;365:1273-83.

34. Johnson GH, Fritsche K. Effect of dietary linoleic acid on markers of inflammation in healthy persons: a systematic review of randomized controlled trials. J Acad Nutr Diet 2012;112:1029-41.

35. Yu Howe-Ming NM, Kalpana S, Weselake Randall J. Bypassing the $\Delta 6$-desaturase enzyme and directly providing $n-3$ and n-6 PUFA pathway intermediates reduces the survival of two human breast cancer cell lines. Eur J Lipid Sci Technol 2015;117:1378-90.

36. Acs B, Pelekanou V, Bai Y, et al. Ki67 reproducibility using digital image analysis: an inter-platform and inter-operator study. Lab Invest 2019;99:107-17.

37. Bankhead P, Loughrey MB, Fernández JA, et al. QuPath: open source software for digital pathology image analysis. Sci Rep 2017;7:16878.

38. Brenna JT, Plourde M, Stark KD, et al. Best practices for the design, laboratory analysis, and reporting of trials involving fatty acids. Am J Clin Nutr 2018;108:211-27.

39. Watson PD, Joy PS, Nkonde C, et al. Comparison of bleeding complications with omega-3 fatty acids + aspirin + clopidogrel-versus--aspirin + clopidogrel in patients with cardiovascular disease. Am J Cardiol 2009;104:1052-4.

40. Eritsland J, Arnesen H, Seljeflot I, et al. Long-Term effects of n-3 polyunsaturated fatty acids on haemostatic variables and bleeding episodes in patients with coronary artery disease. Blood Coagul Fibrinolysis 1995;6:17-22.

41. Knapp HR, Reilly IA, Alessandrini P, et al. In vivo indexes of platelet and vascular function during fish-oil administration in patients with atherosclerosis. N Engl J Med 1986;314:937-42.

42. Ishibashi H, Suzuki T, Suzuki S, et al. Sex steroid hormone receptors in human thymoma. J Clin Endocrinol Metab 2003;88:2309-17.

43. Folch J, Lees M, Sloane Stanley GH. A simple method for the isolation and purification of total lipides from animal tissues. $\mathrm{J} \mathrm{Biol}$ Chem 1957;226:497-509.

44. Field CJ, Ryan EA, Thomson AB, et al. Dietary fat and the diabetic state alter insulin binding and the fatty acyl composition of the adipocyte plasma membrane. Biochem J 1988;253:417-24.

45. Schønberg S, Krokan HE. The inhibitory effect of conjugated dienoic derivatives (CLA) of linoleic acid on the growth of human tumor cell lines is in part due to increased lipid peroxidation. Anticancer Res 1995;15:1241-6.

46. Field CJ, Van Aerde JE, Robinson LE, et al. Effect of providing a formula supplemented with long-chain polyunsaturated fatty acids on immunity in full-term neonates. Br J Nutr 2008;99:91-9.

47. Field CJ, Thomson CA, Van Aerde JE, et al. Lower proportion of CD45R0+ cells and deficient interleukin-10 production by formula-fed infants, compared with human-fed, is corrected with supplementation of long-chain polyunsaturated fatty acids. J Pediatr Gastroenterol Nutr 2000;31:291-9.

48. Richard C, Wadowski M, Goruk S, et al. Individuals with obesity and type 2 diabetes have additional immune dysfunction compared with obese individuals who are metabolically healthy. BMJ Open Diabetes Res Care 2017;5:e000379.

49. Lewis ED, Goruk S, Richard C, et al. Feeding a diet devoid of choline to lactating rodents restricts growth and lymphocyte development in offspring. Br J Nutr 2016;116:1001-12.

50. Thompson FE, Subar AF, Brown CC, et al. Cognitive research enhances accuracy of food frequency questionnaire reports: results of an experimental validation study. J Am Diet Assoc 2002;102:212-25.

51. Subar AF, Thompson FE, Kipnis V, et al. Comparative validation of the Block, Willett, and National Cancer Institute food frequency questionnaires : the Eating at America's Table Study. Am J Epidemiol 2001;154:1089-99.

52. Kipnis V, Subar AF, Midthune D, et al. Structure of dietary measurement error: results of the open biomarker study. Am J Epidemiol 2003;158:14-21.

53. Aaronson NK, Ahmedzai S, Bergman B, et al. The European organization for research and treatment of cancer QLQ-C30: a quality-of-life instrument for use in international clinical trials in oncology. J Natl Cancer Inst 1993;85:365-76.

54. Godin G, Shephard RJ. Godin leisure-time exercise questionnaire. Medicine \& Science in Sports \& Exercise 1997;26:S36-8.

55. Courneya KS, Friedenreich CM. Utility of the theory of planned behavior for understanding exercise during breast cancer treatment. Psychooncology 1999;8:112-22. 